\title{
Ocorrência de muscóideos necrófagos em carcaça de Didelphis albiventris Lund, 1841 (Didelphimorphia, Didelphidae) no Rio Grande do Sul, Brasil
}

\author{
Ândrio Zafalon da Silva* \\ Vanessa Abelaira dos Anjos \\ Paulo Bretanha Ribeiro \\ Rodrigo Ferreira Krüger \\ Departamento de Microbiologia e Parasitologia, Instituto de Biologia \\ Universidade Federal de Pelotas, CEP 96010-900, Pelotas - RS, Brasil \\ *Autor para correspondência \\ azafalon.ib@ufpel.edu.br
}

Submetido em 22/10/2009

Aceito para publicação em 25/02/2010

\section{Resumo}

As informações relativas quanto à similaridade da fauna colonizadora de animais silvestres em decomposição são incipientes entre os diferentes modelos de carcaças. É esperado que espécies que possuam tecidos e dietas alimentares diferentes ao morrerem sejam fontes de colonização de diferentes espécies necrófagas. Para a verificação desta hipótese foi acompanhada a decomposição de uma carcaça de Didelphis albiventris Lund, 1841 (Didelphimorphia, Didelphidae), encontrado morto por atropelamento. Foram criados 2.273 adultos a partir de imaturos coletados na carcaça, representados por espécies pertencentes á Calliphoridae, Muscidae, Sarcophagidae e Fanniidae, com dominância numérica de Lucilia eximia Wiedemann, 1819 (Diptera, Calliphoridae). Outras seis espécies foram encontradas junto de espécimes não identificados de Fanniidae. Todas as espécies encontradas são colonizadoras de modelos tradicionalmente utilizados para estudos de Entomologia Forense.

Unitermos: animais silvestres, Calliphoridae, entomologia forense

\section{Abstract}

Necrophagous Muscoids that develop in carcasses of Didelphis albiventris Lund, 1841 (Didelphimorphia, Didelphidae) in Rio Grande do Sul, Brazil. Information regarding the similarity of the fauna that colonizes decomposing wildlife is insipient among the different carcass models. Species with different tissues and alimentary diets are hypothesized to be a colonization source of different necrophagous species. To verify this hypothesis, we observed the decomposition of a carcass of Didelphis albiventris Lund, 1841 (Didelphimorphia, Didelphidae), that had been run over by a vehicle. 2,273 adults were reared from specimens at immature stages collected on the carcass. The most representative species belongs to Calliphoridae, Muscidae, Sarcophagidae and Fanniidae, with numeric dominance of Lucilia eximia Wiedemann, 1819 (Diptera, Calliphoridae). Another six species were found, besides non-identified Fanniidae specimens. All species were colonizers of traditionally used forensic models.

Key words: Calliphoridae, forensic entomology, wildlife 


\section{Introdução}

Os insetos necrófagos são os primeiros animais a chegar à carcaça para a realização de oviposições e alimentação dos adultos minutos após a morte do animal (Smith, 1986), permanecendo na carcaça ao longo dos estágios de decomposição (Catts e Goff, 1992), portanto assumem fundamental importância para a entomologia forense como uma ferramenta para determinação do IPM (intervalo post-mortem) (Oliveira-Costa, 2003) devido à sucessão faunística das espécies necrófagas, sendo o principal grupo decompositor a família Calliphoridae (Reed, 1958).

A utilização de insetos necrófagos em investigações criminais é bem documentada nos dias atuais (Smith, 1986; Benecke, 1998). Entretanto existem poucas referências que atribuam importância aos insetos em investigações sobre animais vitimados por crimes que acometem a vida silvestre (Watson e Carlton, 2003; Watson e Carlton, 2005).

$\mathrm{Na}$ maioria dos estudos forenses, os modelos animais utilizados são porcos, devido à constituição histológica similar em termos de decomposição aos tecidos de seres humanos. Logo, a fauna colonizadora de cadáveres e carcaças de outros modelos animais poderia ser explicada por esta semelhança (Catts e Goff, 1992).

Além de porcos, já foram utilizados para estudos de comunidades necrófagas: galinhas (Hall e Dotsy, 1993), ratos (Monteiro-Filho e Penereiro, 1987), cachorros (Johnson, 1975), sapos e lagartos (Cornaby, 1974), coelhos (Souza et al., 2008), elefantes (Coe, 1978 apud Smith, 1986) e ursos-negro americanos (Watson e Carlton, 2003; Watson e Carlton, 2005), o que pressupõe diferentes constituições histológicas dos modelos e, por isso, alta variação na diversidade de necrófagos. Além disso, a riqueza de espécies em uma carcaça também pode ser explicada em função da sazonalidade e local de experimentação.

A importância da utilização de insetos necrófagos para fins investigativos encontra-se em solucionar crimes contra a fauna silvestre da região. Portanto, inventariar as espécies que colonizam carcaças, e de acordo com a bibliografia estimar possíveis IPMs a partir do estado de decomposição do animal, fornece suporte aos órgãos fiscalizadores responsáveis pela manutenção do bemestar da fauna silvestre (Watson e Carlton, 2003).

O presente trabalho busca inventariar a fauna de muscóideos necrófagos encontrados em Didelphis albiventris.

\section{Material e Métodos}

O experimento foi realizado em uma área arbórea no campus universitário $\left(31^{\circ} 48^{\prime} 00,31^{\prime}\right.$ 'S, $\left.52^{\circ} 25^{\prime} 04,66^{\prime \prime} \mathrm{W}\right)$ da Universidade Federal de Pelotas, Rio Grande do Sul.

Um cadáver de Didelphis albiventris Lund, 1841 (Didelphimorphia, Didelphidae), provavelmente morto por atropelamento foi encontrado em estrada de acesso ao campus universitário. $\mathrm{O}$ animal com peso de $680 \mathrm{~g}$ não apresentava lesões e foi exposto no ambiente no interior de uma gaiola de metal de dimensões de $90 \mathrm{~cm}$ de comprimento, $70 \mathrm{~cm}$ de largura e $45 \mathrm{~cm}$ de altura, para impedir a interferência de vertebrados necrófagos. A gaiola foi elevada aproximadamente $10 \mathrm{~cm}$ de altura do solo, sustentada pela implantação de dois suportes metálicos. No centro da gaiola foi colocada uma bandeja coletora com serragem (moderadamente úmida) onde a carcaça foi exposta.

A carcaça foi vistoriada diariamente durante 11 dias, do mês de junho de 2008, para coleta de imaturos que foram acondicionados em frascos contendo fígado bovino em decomposição e serragem umedecida, cobertos por gaze para entrada de ar e retenção dos adultos após a emergência. As larvas que já haviam abandonado a carcaça (pré-pupas) foram acondicionadas em frascos com serragem úmida. Em ambas as situações estes frascos foram mantidos em câmara com temperatura variando de $25^{\circ} \mathrm{C} \pm 2^{\circ} \mathrm{C}$, umidade relativa do ar acima de $75 \%$ e fotofase com cerca de $12 \mathrm{~h}$.

Os adultos que emergiram foram sacrificados com acetato de etila e armazenados em álcool à $70^{\circ} \mathrm{GL}$ para posterior identificação. A identificação dos muscóideos adultos baseou-se nas chaves propostas por Carvalho e Ribeiro (2000), Carvalho e Couri (2002) e Carvalho et al. (2002). 


\section{Resultados}

Foram criados 2273 dípteros adultos a partir de imaturos coletados na carcaça de D. albiventris. Estes indivíduos representaram as famílias Calliphoridae, Muscidae, Sarcophagidae e Fanniidae (Tabela 1), com maior abundância das espécies Lucilia eximia Wiedemann, 1819 (Calliphoridae) e Hemilucilia segmentaria Fabricius, 1805 (Calliphoridae). A família Sarcophagidae foi a segunda mais abundante e representada por Sarcophaga (Liopygia) crassipalpis Macquart, 1839.

As espécies de Muscidae, Psilochaeta pampiana Shannon \& Del Ponte, 1926, Stomoxys calcitrans Linnaeus, 1758, Muscina stabulans Fallén, 1817 e Musca domestica Linnaeus, 1758 representaram aproximadamente de $5,55 \%$. Os faniídeos foram pouco representativos na totalidade de dípteros capturados.

TABELA 1: Dípteros necrófagos obtidos em cadáver de Didelphis albiventris, no outono, no municipio do Capão do Leão, RS.

\begin{tabular}{lcc}
\hline \multicolumn{1}{c}{ Táxons } & $\begin{array}{c}\text { Frequência } \\
\text { Absoluta }\end{array}$ & $\begin{array}{c}\text { Frequência } \\
\text { Relativa (\%) }\end{array}$ \\
\hline Calliphoridae & $\mathbf{1 8 7 4}$ & $\mathbf{8 2 , 4 5}$ \\
Lucilia eximia & 1473 & 64,80 \\
Hemilucilia segmentaria & 401 & 17,60 \\
Muscidae & $\mathbf{1 2 6}$ & $\mathbf{5 , 5 5}$ \\
Psilochaeta pampiana & 65 & 2,85 \\
Stomoxys calcitrans & 50 & 2,19 \\
Muscina stabulans & 9 & 0,39 \\
Musca domestica & 2 & 0,08 \\
Sarcophagidae & $\mathbf{2 6 7}$ & $\mathbf{1 1 , 7 4}$ \\
Sarcophaga (Liopygia) & 267 & 11,74 \\
crassipalpis & & $\mathbf{0 , 2 6}$ \\
Fanniidae & $\mathbf{6}$ & $\mathbf{1 0 0 , 0 0}$ \\
Total & $\mathbf{2 2 7 3}$ &
\end{tabular}

\section{Discussão}

As espécies encontradas em $D$. albiventris foram observadas em outros modelos animais como coelho (Souza et al., 2008) e porco (Centeno et al., 2002) provavelmente por causa da dominância ecológica de Calliphoridae existente nestes sistemas. Normalmente, ocorre a dominância de até três espécies nestes recursos efêmeros como carcaças de porco (Carvalho e Linhares,
2001; Moura, 2004) e cadáveres humanos (OliveiraCosta et al., 2001).

Souza et al. (2008) observaram que L. eximia junto de Chrysomya albiceps Wiedemann, 1819 (Diptera, Calliphoridae) são os principais indicadores forense para determinação do intervalo postmortem (IPM) para a região sul do Brasil. Isto se deve a não preferência quanto à estação do ano, sendo observadas em todos os estágios de decomposição e indicadoras do IPM mínimo. Nesta mesma região, H. segmentaria foi considerada um bom indicador do IPM para o outono (Souza et al., 2008).

Watson e Carlton $(2003 ; 2005)$ observaram um padrão similar de dominância ecológica de Calliphoridae entre carcaças de animais silvestres como ursos-negro americano, crocodilos, veados da cola branca e modelos tradicionalmente utilizados como suínos. Talvez, seja mais importante o tempo de exposição e massa corpórea da carcaça, condição climática e região geográfica do que o modelo utilizado.

As espécies de Muscidae encontradas, $M$. domestica e S. calcitrans, são fortemente associadas ao ambiente antrópico e utilizam diversos substratos em decomposição para o desenvolvimento de seus imaturos (Skidmore, 1985). Estas espécies foram encontradas em carcaça de porco (Salviano et al., 1996) enquanto exemplares de $M$. domestica foram coletadas por Monteiro-Filho e Penereiro (1987) em ratos, e por Oliveira-Costa (2005) em cadáveres humanos no Rio de Janeiro.

Já M. stabulans só encontra registro como espécie de importância forense em estudos na Europa (Grassberger e Frank, 2004), Argentina (Centeno et al., 2002) e no sul do Brasil (Souza et al., 2008).

A $P$. pampiana foi a espécie com maior abundância entre os muscídeos. Esta espécie já havia sido observada visitando carcaças de coelhos (A. S. B. Souza - dados não publicados) e ratos, associada a ambiente florestado, com preferência sazonal pelo outono e pelo inverno (Moura et al., 1997). Esta ocorrência é o primeiro registro de desenvolvimento desta espécie em carcaças, o que leva a crer que possua maior valor para as estimativas de IPM.

Sarcophaga (L.) crassipalpis é uma indicadora do IPM em cadáveres humanos (Nishida, 1984) e sua ocorrência em carcaça de $D$. albiventris é o primeiro registro de colonização deste substrato no Brasil. 


\section{Agradecimentos}

Agradecemos o Biólogo Juliano Lessa Pinto pela revisão do abstract e a Prof ${ }^{\mathrm{a}}$. $\mathrm{Dr}^{\mathrm{a}}$. Gertrud Muller pelo recolhimento da carcaça de $D$. albiventris.

\section{Referências}

Benecke, M. 1998. Six forensic entomology cases: Description and commentary. Journal of Forensic Science, 43 (4): 797-805.

Carvalho, C. J. B. De; Couri, M. S. 2002. Part I. Basal Groups. In: Carvalho, C. J. B. De (Ed.). Muscidae (Diptera) of the Neotropical Region: Taxonomy. Ed. UFPR, Curitiba, Brasil, 287pp.

Carvalho, C. J. B. De; Moura, M. O.; Ribeiro, P. B. 2002. Chave para adultos de dípteros (Muscidae, Fanniidae, Anthomyiidae) associados ao ambiente humano no Brasil. Revista Brasileira de Entomologia, 46 (2): 107-114.

Carvalho, C. J. B. De; Ribeiro, P. B. 2000. Chave de identificação das espécies de Calliphoridae (Diptera) do sul do Brasil. Revista Brasileira de Parasitologia Veterinária, 9 (2): 169-173.

Carvalho, L. M. L.; Linhares, A. X. 2001. Seasonality of insects succession and pig carcass decomposition in a natural forest área in southearstern Brazil. Journal of Forensic Science, 46: 604-608.

Catts, E. P.; Goff, M. L. 1992. Forensic entomology in criminal investigations. Annual Review of Entomology, 37: 253-272.

Centeno, N.; Maldonado, M; Oliva, A. 2002. Seasonal of arthropods occuring on sheltered an unsheltered pig carcass in Buenos Aires province (Argentina). Forensic Science International, 126: 6370 .

Cornaby, B. W. 1974. Carrion reduction by animals in constrasting tropical habitats. Biotropica, 6: 51-63.

Grassberger, M.; Frank, C. 2004. Intial study of arthropod succession on pig carrion in a central european urban habitat. Journal of Medical Entomology, 41: 511- 523.

Hall, R. D.; Dotsy, K. E. 1993. Length of time after death: Effect on attraction and oviposition or larviposition of midsummer blowflies (Diptera, Calliphoriadae) and the flesh flies (Diptera, Sarcophagidae) of medical importance in Missouri. Annals of entomology society of America, 86: 589-593.

Johnson, M. D. 1975. Seasonal and Microseral variations in the insect populations on carrion. American Midland Naturalist, 93: 79-90.
Monteiro-Filho, E. L. A.; Penereiro, J. L. 1987. Estudo de decomposição e sucessão sobre uma carcaça animal numa área do estado de São Paulo, Brasil. Revista Brasileira de Biologia, 47: 289-295.

Moura, M. O. 2004. Variação espacial como promotor da coexistência em comunidades de insetos necrófagos. Revista Brasileira de Zoologia, 21 (3): 409-419.

Moura, M. O.; Carvalho, C. J. B. De; Monteiro-Filho, E. L. A. 1997. A preliminary analysis of insects of medico-legal importance in Curitiba, state of Paraná. Memórias do Instituto Oswaldo Cruz, 92 (2): 269-274.

Nishida. K. 1984. Experimental studies on the estimation of postmortem intervals by means of fly larvae infesting human cadavers. Japanese Journal of Forensic Medicine, 38: 24-41.

Oliveira-Costa, J. 2005. Levantamento da entomofauna cadavérica com vistas à formação de um banco de dados de aplicação em investigações de morte violenta do estado do Rio de Janeiro. Tese de Doutorado, Universidade Federal do Rio de Janeiro, UFRJ, Brasil, 133pp

Oliveira-Costa, J.; Meloo-Patiu, C. A.; Lopes, S. M. 2001. Dípteros muscoideos associados com cadáveres humanos no local da morte, no estado do Rio de Janeiro, Brasil. Boletim do Museu Nacional, Série Zoologia, 470: 1-10.

Reed, H. B. Jr. 1958. A study of dog carcass communities in Tennessee, with special reference to the insects. The American Midland Naturalist Journal, 59: 213-245.

Salviano, R. J. B.; Mello, R. P.; Beck, R. F. S.; Ferreira, A. 1996. Calliphoridae (Diptera) associated with human corpses in Rio de Janeiro, Brazil. Entomologia y Vectores, 3: 145-146.

Skidmore, P. 1985. The biology of the Muscidae of the world. Series Entomologica. Junk, Dordrecht, Netherlands, 550pp.

Smith, K. G. V. 1986. A Manual of Forensic Entomology. Cornell University Press, Ithaca, USA, 205pp.

Souza, A. S. B.; Kirst, F. D.; Krüger, R. F. 2008. Insects of forensic importance from Rio Grande do Sul state in southern Brazil. Revista Brasileira de Entomologia, 52 (4): 641-646.

Watson, E. J.; Carlton, C. E. 2003. Spring succession of necrophilous insects on wildlife carcasses in Louisiana. Journal of Medical Entomology, 40: 338-347.

Watson, E. J.; Carlton, C. E. 2005. Insect succession and decomposition of wildlife carcasses during fall and winter in Louisiana. Journal of Medical Entomology, 42 (2): 193-203. 